\title{
The use of multi-frequency impedimetry for the monitoring of chronic wounds: the BIPPED clinical study
}

\author{
Marie Muller ${ }^{1}$, Justine Cristante ${ }^{1}$, Alison Foote ${ }^{2}$, Amalric Montalibet ${ }^{3}$, Sadok Gharbi4, \\ Eric McAdams ${ }^{3}$, Pascale Pham ${ }^{4}$ \\ ${ }^{1}$ Grenoble University Hospital, Department of Endocrinology, Grenoble, F-38000 France \\ ${ }^{2}$ Grenoble University Hospital, Clinical Research Centre, INSERM CIC 1406, Grenoble, F-38000 France \\ ${ }^{3}$ Université de Lyon, Institute des Nanotechnologies de Lyon, INL-UMR5270, CNRS, INSA Lyon, Villeurbanne, F- \\ 69100, France \\ ${ }^{4}$ CEA-Leti, Univ. Grenoble-Alpes, Minatec Campus 38054 Grenoble, France \\ (MMuller@chu-grenoble.fr; JCristante@chu-grenoble.fr; AFoote@chu-grenoble.fr; \\ amalric.montalibet@insa-lyon.fr; sadok.gharbi@cea.fr; eric.mcadams@insa-lyon.fr; \\ pascale.pham@free.fr)
}

\begin{abstract}
The number of people developing type II diabetes is verging on epidemic proportions in some countries. Along with an aging population, the incidence of chronic diabetic foot ulcers and venous leg ulcers is increasing and threatens to pose a problem for healthcare systems. A smart, wearable autonomous wound therapy and monitoring device is the goal of the Swan-iCare project, which involves 9 consortium members and is funded by the European commission under the FP7 program. The device is intended to shorten hospital stays by enabling patients to return home and have their wounds remotely monitored by the clinician. A miniaturized, bio-impedance sensor system is among the sensors under development. In this paper we describe the promising preliminary results of a clinical study on the use of bioimpedance measurements to monitor the healing processes of diabetic foot ulcers.
\end{abstract}

\section{General Terms}

Experimentation, Human Factors,

\section{Keywords}

Diabetic foot ulcers, bioelectrical impedimetry, remote monitoring, bio-electrical impedance

\section{INTRODUCTION}

Chronic wounds represent a major and growing burden for healthcare systems throughout the world.

As a consequence, recent developments in healthcare policies encourage clinicians to use remote monitoring in the follow-up of chronic wounds (diabetic foot ulcers (DFU), venous leg ulcers
(VLU), pressure ulcers, etc.), so as to improve care, reduce time spent in hospital and reduce healthcare costs.

However, at present, clinicians encounter a lack of objective data to enable the effective monitoring of patients' clinical conditions at home, and also a lack of validated monitoring tools.

Much progress has been made in the understanding of chronic ulcer pathophysiology, such as the key role of matrix metalloproteinases (proteolytic enzymes responsible for the degradation of the extra cellular matrix) and growth factors. The wound $\mathrm{pH}$ is also thought to vary according to the healing phase, or in the presence of wound infection. It is suggested that Bioelectrical Impedance Spectroscopy (BIS) could also be a good indicator of the cellular processes of healing [1] and that BIS parameters could potentially form the basis of a novel remote wound monitoring system. This could be implemented at the patient's home by a nurse, or autonomously provide measurements several times a day, with data transferred to the clinician in charge of the patient. Under alternating electrical excitation biological tissue has complex bioelectrical impedance which depends on its composition and the frequency of the very low amplitude (microAmps) applied alternating current. This behavior is usually represented on Nyquist plots (-XS vs RS) [2].

This paper describes preliminary results for a miniaturized impedimetric sensor system being developed as part of the SwaniCare (Smart wearable and autonomous negative pressure device for wound monitoring and therapy) project, an EC funded project (FP7-ICT Project No. 317894) whose overall goal is to develop a wearable, negative-pressure wound therapy and monitoring device equipped with a range of sensors and ICT to provide continuous real time data to the medical support team.

\section{METHODS}

The BIPPED (BIoImpédance sur les Plaies chroniques des PiEDs) study is an experimental clinical research study approved by our local ethics committee. BIPPED's main objective is to identify the impedimetric parameters of hard-to-heal ulcers that correlate with the severity of the wound, its size and the phase of healing. 
We are following 20 diabetic patients presenting with a lower limb ulcer, aged 18 to 85 , over 3 months with 5 impedance measurements (day 1 , day 5 , day 30 , day 60 and day 90). Written informed consent is collected for each patient. Patients with a second ulcer on the opposite lower limb are excluded, as well as those suffering from unilateral oedema or presenting with any contra-indication to impedance measurements (pacemaker etc.).

At each visit, clinical parameters are registered (wound depth, size and appearance, presence of fibrin or granulation tissue buds, glycemia, digital oxygen saturation etc.). Impedance measurements are performed using a commercial bio-impedance device designed for whole-body in vivo BIA measurements - for the estimation of body composition - using a 4-electrode technique (SFB7 Impedimed ${ }^{\mathrm{TM}}$ ) within the $1 \mathrm{kHz}$ to $1 \mathrm{MHz}$ frequency range. A standardized protocol is followed and impedance measurements take in total around 15 minutes at each visit (see Figure 1).
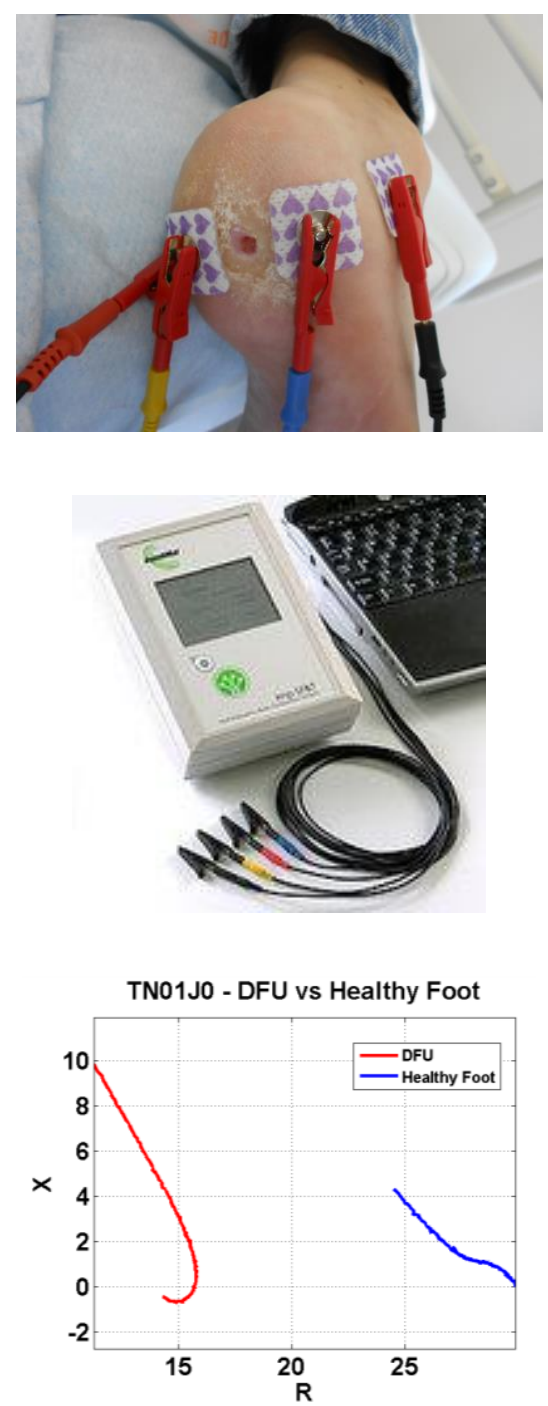

Figure 1: Overview of the BIPPED protocol: 4 point impedance measurement (top) on a Diabetic Foot Ulcer,

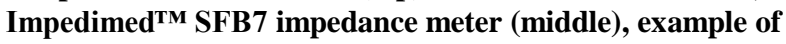
measured Nyquist plots of DFU (red) and the healthy foot (blue) (bottom).

\section{INITIAL RESULTS}

Nyquist plots of the impedance data for 9 patients and 2 controls (healthy volunteers) show that the impedances of the foot with the chronic wound (DFU) is distinguishable from that of the healthy foot (HF) (see Figure 2). This result has to be confirmed using statistical analysis over the 20 patients to be included in the BIPPED clinical study.

\section{DISCUSSION}

Initial results from the BIPPED study clearly show a significant difference between the impedances obtained on a foot with a wound compared to those on the patient's healthy foot, with the impedance of the wound being smaller than that of the healthy foot. Significantly, the differences between the impedances of the feet of the controls were much smaller than those for the diabetic patients.

The results generally evidence segments of the anticipated depressed semi-circular arc loci on the Nyquist plots [2]. However, the presence in many cases of significant loci distortions, due to a combination of factors [3], highlights the need for the design of more appropriate measurement electronics specifically for localized measurements and a custom-designed, high-performance electrode array for accurate, optimally targeted diabetic foot wound assessment.

The role of various biomedical sensors in the monitoring of wound healing has been recently highlighted in a review by Salvo et al [4]. Many promising sensors will probably emerge within the next few years. However, these sensors will have to be economically attractive, and compatible with mass production. Impedance measurements on and around chronic ulcers most probably fulfill these criteria, and have already been tested in the prevention of pressure ulcers by Maharbiz et al [5]. These authors demonstrated on animal models that impedance measurements are strongly correlated with tissue health, and with the risk of developing ulcers as a result of prolonged pressure applied to the skin. These results open the way to the development of "smart" dressings incorporating electrodes in contact with the skin, capable of measuring the electrical impedance of intact skin and determining its vitality.

Regarding chronic wounds, however, there is still a lack of impedance measurement data on wounds during the healing process. The link between such measurements and the evolution of wound healing needs to be formally established.

\section{CONCLUSION}

Our initial experimental data are encouraging and we believe that our study will furnish new insight into the pathophysiology of chronic wounds. Our preliminary results suggest the potential feasibility of using multifrequency impedimetric measurements for remote monitoring of chronic wound healing, This should help enable the clinician to follow the wound healing process at a distance and enable patients with chronic wounds to return home, reducing the need for prolonged hospitalization and/or frequent visits to the clinic. 


\section{DFU vs HF}
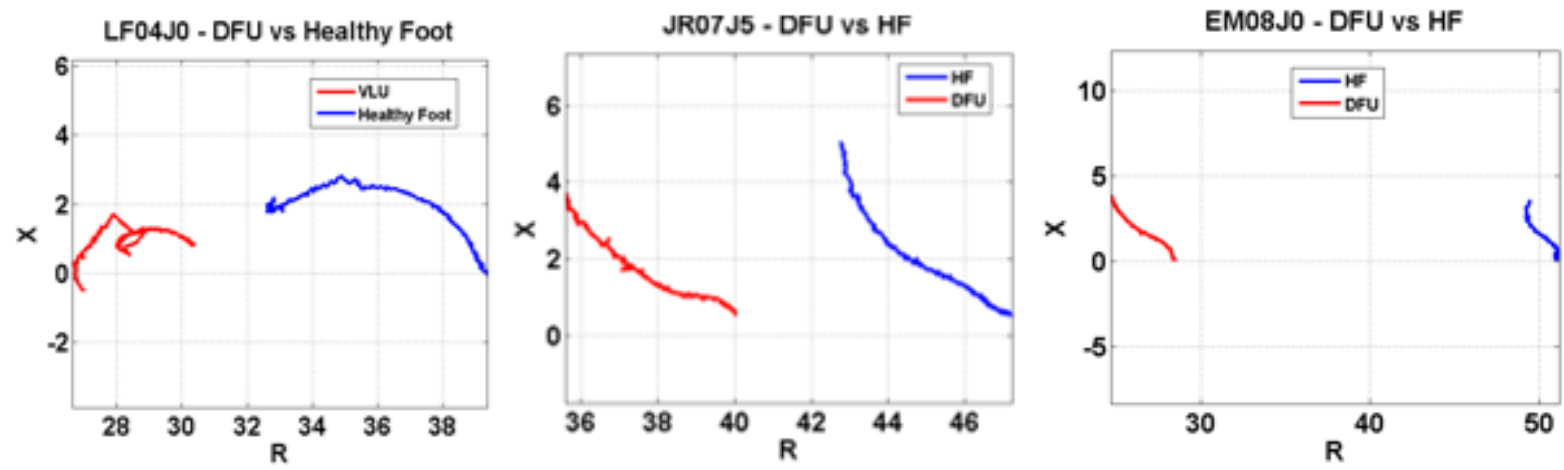

\section{Controls (Left Foot, Right Foot)}
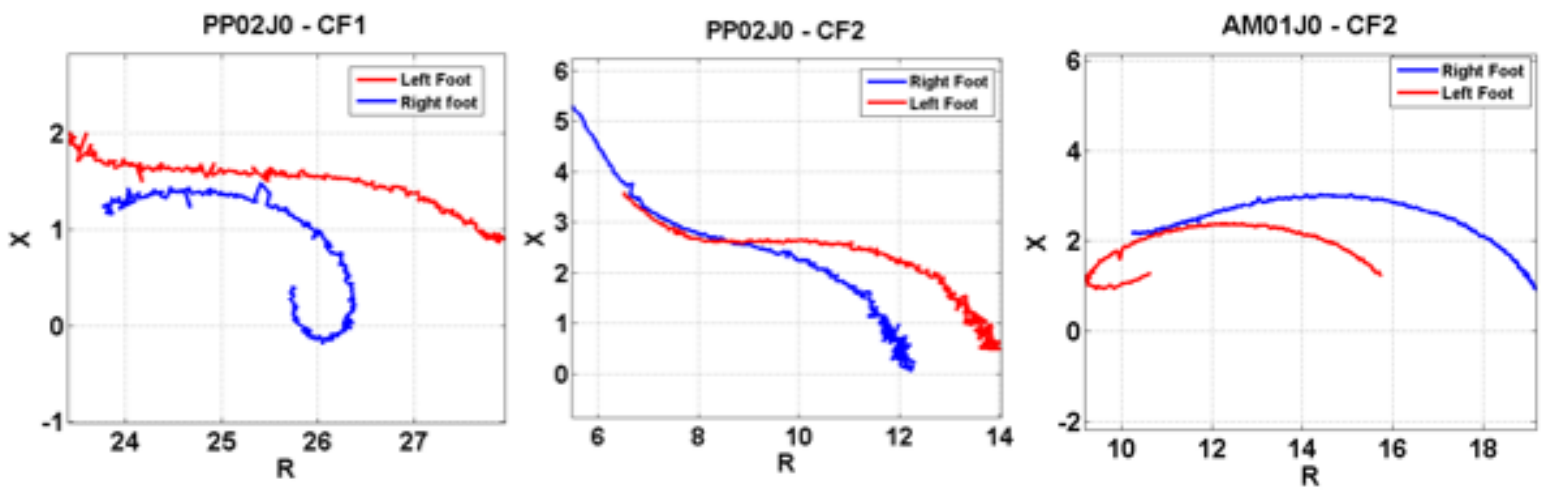

Figure 2: Top: Nyquist Plots on 3 diabetic patients with chronic wounds on one foot: DFU (red), comparison between the DFU (red) and the healthy foot (blue). Bottom: Nyquist plots on 2 healthy volunteers, comparison between the left foot (red) and the right foot (blue).

\section{REFERENCES}

[1] Lukaski, H.C. and Moore, M. 2012. Bioelectrical impedance assessment of wound healing. J. Diabetes Sci. Technol., 6, 1, 209-212.

[2] McAdams, E.T. 2006. Bioelectrodes, In Encyclopedia of Medical Devices and Instrumentation, 2nd Edition, Ed John G Webster, Wiley InterScience, ISBN 0-471-26358-3, 120-166.

[3] Bogónez-Franco, P., Pham, P. C. Gehin, C., B. Massot, B. Delhomme, G., Guillemaud, R., McAdams, E. Effect of electrode contact impedance mismatch on 4-electrode measurements of small body segments using commercial BIA devices. In 20th IMEKO TC4 International Symposium and 18th International Workshop on ADC Modelling and Testing Research on Electric and Electronic Measurement for the Economic Upturn, Benevento, Italy. September 15-17, 2014.
[4] Salvo, P., Dini, V., Di Francesco, F., M. Romanelli, M. 2015. The role of biomedical sensors in wound healing. Wound Medicine." 8 (April), 15-18.

[5] Swisher, S.L., Lin, M.C., Liao A., Leeflang, E.J., Yasser Khan, Y., Pavinatto, F.J., Mann,K., Naujokas, A., Young, D., Roy,S., Harrison, M.R., Arias, A.C.,Vivek Subramanian, V., Maharbiz, M.M. 2015. Impedance sensing device enables early detection of pressure ulcers in vivo. Nature Communications 6, Article number: 6575. 